\title{
Some Nonlinear Aspects of Wind-Wave Interactions ${ }^{1,2}$
}

\author{
F. A. LEE \\ Dept. of Oceanography, University of Washington, Seattle 98105
}

(Manuscript received 31 January 1972, in revised form 12 June 1972)

\begin{abstract}
Nonlinear momentum transfer from wind to wave has been studied on the basis of a perturbation expansion procedure. Two nonlinear effects are presented, namely the generation of higher harmonics of the disturbances and the mean wind profile modification by Reynolds stress. The second harmonic Reynolds stress is found to be $2(k a)^{2}$ times the first harmonic Reynolds stress, where $k$ is the wavenumber and $a$ the wave amplitude. To the order of $a^{2}$, the wind profile is modified by the Reynolds stress of the first harmonic.
\end{abstract}

\section{Introduction}

When a turbulent air stream blows over water, it generates waves that grow with time and fetch. These wind waves are able to extract energy from the mean wind field, provided that the Reynolds stresses in the airflow associated with the wind wave are positive. The energy can then be passed on to the sea wave by the action of pressure forces at the oscillating surface.

Studies of wind-wave interaction are accumulating on both theoretical and experimental fronts: notably the works by Phillips (1957) and Miles (1957) on the theoretical side; and Stewart (1970), Shemdin (1969), and Dobson (1971) on laboratory experiments and open sea measurements. Both laboratory experiments and open sea measurements suggest that Miles' inviscid theory substantially underpredicts the energ $y^{-}$transfer. More recently, Reynolds (1968) formulated the problem by introducing an eddy viscosity coefficient to relate the turbulent stresses perturbed by wave motion to the perturbation strain rates. Calculations were carried out for the wind profile with wavenumber and wave speeds corresponding to four sets of Stewart's experiment. The phases of $u$ and $w$ (measured with respect to the surface wave crests) are in good agreement, while the measured amplitudes are low by significant factors. Stewart finds that the energetic content of the second harmonic is approximately half that of the fundamental, and the calculations made by Reynolds suggest that nonlinear effects may account for the striking differences between the linear theory and the measurements.

\footnotetext{
${ }^{1}$ Presented at the Conference on the Interaction of the Sea and the Atmosphere, 1-3 December 1971, Ft. Lauderdale, Fla.

${ }^{2}$ Contribution No. 660, Department of Oceanography, University of Washington.
}

It is the purpose of this study to assess the nonlinear effects. The model under consideration is an extension of Reynolds' linear model to the nonlinear domain by taking account of the higher order terms. The net contribution of momentum transfer to a propagating sinusoidal wave will be investigated by considering wave-related perturbation stresses in the airflow predicted by this model. The water surface is, therefore, assumed to respond to turbulent eddies impinging on it.

\section{Formulation}

The turbulent velocity field was decomposed into a mean component $\overrightarrow{\mathrm{U}}$, a wave-induced component $\vec{U}$, and a random component $\vec{u}^{\prime}$ representing background turbulence:

$$
\vec{u}=\overrightarrow{\mathbf{U}}+\vec{U}+\vec{u}^{\prime}
$$

Quantities ${ }^{3}$ will be presumed normalized on suitable reference velocity and length scales $\left(\mathbf{U}_{r}\right.$ and $\left.\delta\right)$. The equations will be restricted to incompressible flow with constant viscosity, and to flows that are statistically periodic in time. The water surface will be assumed to respond to turbulent eddies impinging on it. The net contribution of the turbulent eddies on momentum transfer to a propagating sinusoidal wave will be investigated. The water surface will be assumed to be composed of a sinusoidal component

$$
\zeta=a \cos k(x-C t) \text {. }
$$

The velocity field will be considered in a frame of reference traveling with speed $C$ in the positive $x$ direction. The wave-induced velocity components become stationary and sinusoidal in $x$. The velocity

\footnotetext{
${ }^{3} \mathrm{~A}$ list of svmbols is given in the Appendix.
} 
field is specified by

$$
\left.\begin{array}{rrr}
u & =\mathbf{U}(z)-C+U(x, z)+u^{\prime}(x, y, z, t) \\
v & = & v^{\prime}(x, y, z, t) \\
w & = & W(x, z)+w^{\prime}(x, y, z, t)
\end{array}\right\} .
$$

Turbulence is homogeneous in the $y$ direction and the wave grows in the $x$ direction. In order to extract the organized motion from a background field of turbulent motion, we introduce a time average

$$
U(\vec{x})=\lim _{T \rightarrow \infty} \frac{1}{T} \int_{0}^{\infty} u(\vec{x}, t) d t
$$

and a phase average

$$
\langle U(x, t)\rangle=\lim _{N \rightarrow \infty} \frac{1}{N} \sum_{n=0}^{N} u(\vec{x}, t+n \tau),
$$

where $\tau$ is the period of the wave. The organized wave is defined by $U=\langle U(\vec{x}, t)\rangle-U(\vec{x})$. By making use of the time and phase averages, one may develop the equations the mean field, the organized wave, and the turbulence as follows:

$$
\begin{aligned}
& \frac{\partial \mathrm{U}}{\partial x}=0 \\
& \frac{\partial U}{\partial x}+\frac{\partial W}{\partial z}=0 \\
& \frac{\partial u^{\prime}}{\partial x}+\frac{\partial v^{\prime}}{\partial y}+\frac{\partial w^{\prime}}{\partial z}=0 \\
& (\mathbf{U}-C) \frac{\partial U}{\partial x}+W \frac{\partial \mathbf{U}}{\partial z}+\frac{\partial}{\partial x}\left(U^{2}\right)+\frac{\partial}{\partial z}(U W) \\
& +\frac{\partial}{\partial x}\left\langle u^{\prime 2}\right\rangle+\frac{\partial}{\partial z}\left\langle u^{\prime} w^{\prime}\right\rangle \\
& =-\frac{1}{\rho} \frac{\partial}{\partial x}(P+p)+\nu\left(\frac{\partial^{2}}{\partial x^{2}}+\frac{\partial^{2}}{\partial z^{2}}\right)(\mathbf{U}+U) \\
& \frac{\partial}{\partial x}\left\langle u^{\prime} v^{\prime}\right\rangle+\frac{\partial}{\partial z}\left\langle v^{\prime} w^{\prime}\right\rangle=0 \\
& (\mathbf{U}-C) \frac{\partial W}{\partial x}+\frac{\partial}{\partial x}(U W)+\frac{\partial}{\partial z}\left(W^{2}\right)+\frac{\partial}{\partial x}\left\langle u^{\prime} w^{\prime}\right\rangle \\
& +\frac{\partial}{\partial z}\left\langle w^{\prime 2}\right\rangle=-\frac{1}{\rho \partial z}(P+p)-g+\nu\left(\frac{\partial^{2}}{\partial x^{2}}+\frac{\partial^{2}}{\partial z^{2}}\right) W
\end{aligned}
$$

Averaging in the $x$ direction yields the linearized disturbance equations

$$
\begin{aligned}
& (\mathbf{U}-C) \frac{\partial U}{\partial x}+W \frac{\partial \mathbf{U}}{\partial z}+\frac{\partial}{\partial x}\left(U^{2}\right)+\frac{\partial}{\partial z}(U W-\overline{U W}) \\
& \left.+\frac{\partial}{\partial x}\left\langle u^{\prime 2}\right\rangle+\frac{\partial}{\partial z}\left(\left\langle u^{\prime} w^{\prime}\right\rangle-\overline{\left\langle u^{\prime} w^{\prime}\right.}\right\rangle\right) \\
& =-\frac{1 \partial p}{\rho \partial x}+\nu\left(\frac{\partial^{2} U}{\partial x^{2}}+\frac{\partial^{2} U}{\partial z^{2}}\right) \\
& (\mathbf{U}-C) \frac{\partial W}{\partial x}+\frac{\partial}{\partial x}(U W)+\frac{\partial}{\partial z}\left(W^{2}-\overline{W^{2}}\right) \\
& +\frac{\partial}{\partial x}\left\langle u^{\prime} w^{\prime}\right\rangle+\frac{\partial}{\partial z}\left(\left\langle w^{\prime 2}\right\rangle-\overline{\left.\left\langle w^{\prime 2}\right\rangle\right)}\right. \\
& \left.=-\frac{1 \partial W}{\rho \partial z}+\nu\left(\frac{\partial^{2} W}{\partial x^{2}}+\frac{\partial^{2} W}{\partial z^{2}}\right)\right)
\end{aligned}
$$

All wave-induced perturbation quantities are assumed to have the form

$$
q=\hat{q}(z) e^{i k(x-C t)} .
$$

The equations of motion (6) become

$$
\left.\begin{array}{rl}
i k(\mathbf{U}-C) \hat{U}+\hat{W} \frac{\partial \mathbf{U}}{\partial z}= & -\frac{i k}{\rho} \hat{p}-i k\left(\hat{r}_{11}+\hat{s}_{11}\right) \\
& -\frac{\partial}{\partial z}\left(\hat{r}_{13}+\hat{s}_{13}\right)+\nu\left(\frac{\partial^{2} \hat{U}}{\partial z^{2}}-k^{2} \hat{U}\right) \\
i k(\mathbf{U}-C) \hat{W}=-\frac{1 \partial \hat{p}}{\rho \partial z}-i k\left(\hat{r}_{13}+\hat{s}_{13}\right) \\
-\frac{\partial}{\partial z}\left(\hat{r}_{33}+\hat{s}_{33}\right)+\nu\left(\frac{\partial^{2} \hat{W}}{\partial z^{2}}-k^{2} \hat{W}\right)
\end{array}\right\}
$$

With the introduction of a streamfunction $\psi$ defined by

$$
\left.\begin{array}{c}
U=\frac{\partial \psi}{\partial z}, \quad \hat{U}=\frac{\partial \phi}{\partial z} \\
W=-\frac{\partial \psi}{\partial x}, \quad \hat{W}=-i k \phi
\end{array}\right\},
$$

we may combine the two equations of motion into a single equation

$$
\begin{aligned}
& (\mathbf{U}-C)\left(\frac{\partial^{2}}{\partial z^{2}}-k^{2}\right) \phi-\phi \frac{\partial^{2} \mathbf{U}}{\partial z^{2}}=\frac{\nu}{i k}\left(\frac{\partial^{2}}{\partial z^{2}}-k^{2}\right)^{2} \phi \\
& +\frac{i}{k}\left(\frac{\partial^{2}}{\partial z^{2}}+k^{2}\right)\left(\hat{r}_{13}+\hat{s}_{13}\right)-\frac{\partial}{\partial z}\left(\hat{r}_{11}+\hat{s}_{11}-\hat{r}_{33}-\hat{s}_{33}\right),
\end{aligned}
$$


where $r_{i j}$ are stress terms perturbed by wave-induced motion and $s_{i j}$ are stress terms perturbed by turbulent motion. Reynolds (1968) assumed that all $r_{i j}$ terms were small compared to $s_{i j}$ terms. Then Reynolds related the perturbation stresses $s_{i j}$ to the perturbation strains $S_{i j}$

$$
s_{i j}=-2 E S_{i j}
$$

where

$$
S_{i j}=\frac{1}{2}\left(\frac{\partial U_{i}}{\partial x_{j}}+\frac{\partial U_{j}}{\partial x_{i}}\right)
$$

and $E$ is a function of $z$. If the eddy viscosity $E$ is set equal to zero and the Reynolds number $R e$ is set equal to infinity, Eq. (10) reduces to a Rayleigh equation, which was the basis considered by Miles. With all the terms expressed in terms of $\phi$, by expansion in terms of perturbation $k a, \phi=\phi_{1}+k a \phi_{2}+\cdots$, we have

$$
\begin{gathered}
(\mathbf{U}-C)\left(\frac{d^{2} \phi_{1}}{d z^{2}}-k^{2} \phi_{1}\right)-\phi_{1}-\frac{d^{2} \mathbf{U}}{d z^{2}} \\
=\frac{1}{i k \operatorname{Re}}(1+E)\left(\frac{d^{2}}{d z^{2}}-k^{2}\right)^{2} \phi_{1} \\
+\frac{2}{i k \operatorname{Re}} \frac{d E}{d z}\left(\frac{d^{3} \phi_{1}}{d z^{3}}-k^{2}-\frac{d \phi_{1}}{d z}\right) \\
+\frac{d^{2} E / d z^{2}}{i k \operatorname{Re}}\left(\frac{d^{2} \phi_{1}}{d z^{2}}+k^{2} \phi_{1}\right),
\end{gathered}
$$

where $\phi_{1}$ refers to first-order streamfunction. Similarly, we obtain the equation governing the second harmonics $\phi_{2}$ by

$$
\begin{gathered}
(\mathbf{U}-C)\left(\frac{d^{2}}{d z^{2}}-4 k^{2}\right) \phi_{2}-\frac{d^{2} \mathbf{U}}{d z^{2}} \phi_{2} \\
=\frac{1}{2 i k \operatorname{Re}}(1+E)\left(\frac{d^{2}}{d z^{2}}-4 k^{2}\right)^{2} \phi_{2} \\
+\frac{1}{i k \operatorname{Re} d z} \frac{d E}{d z}\left(\frac{d^{3} \phi_{2}}{d z^{3}}-k^{2} \frac{d \phi_{2}}{d z}\right) \\
+\frac{1}{2 i k \operatorname{Re} \frac{d^{2} E}{d z^{2}}\left(\frac{d^{2}}{d z^{2}}+4 k^{2}\right) \phi_{2}} \\
+\frac{1}{2}\left(\frac{d \phi_{1}}{d z} \frac{d^{2} \phi_{1}}{d z^{2}}-\phi_{1} \frac{d^{3} \phi_{1}}{d z^{2}}\right)
\end{gathered}
$$

In order to simplify the boundary conditions, we adopt the curvilinear coordinates suggested by Benjamin (1959),

$$
\left.\begin{array}{c}
\xi=x-i a e^{-k(z-i x)} \\
\eta=z-a e^{-k(z-i x)}
\end{array}\right\}
$$

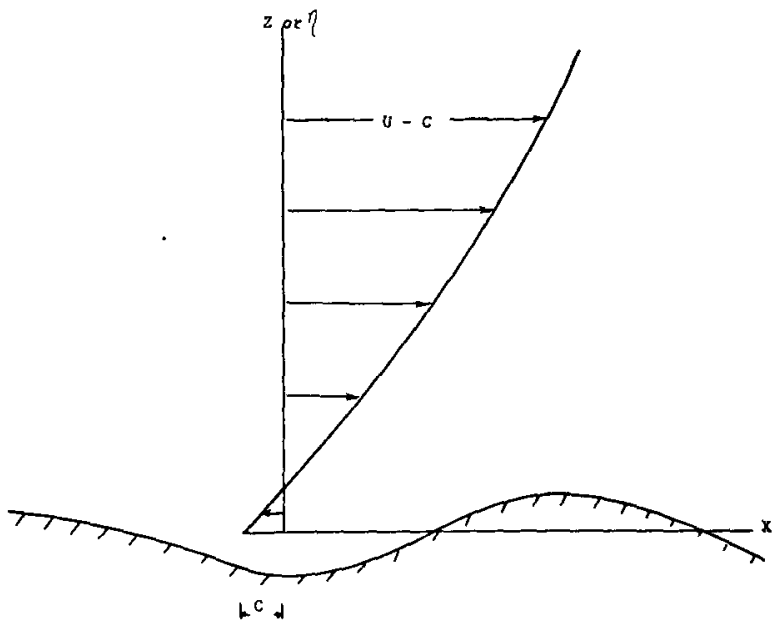

FIG. 1. Undisturbed velocity profiles as it would appear to an observer moving with the wave.

shown in Fig. 1. The wind-wave interaction problem can finally be formulated in terms of the following boundary value problems:

\section{First order}

$$
\begin{aligned}
(\mathrm{U}-C)\left(\boldsymbol{\phi}_{1}{ }^{\mathrm{II}}-k^{2} \phi_{1}\right)-\mathrm{U}^{\mathrm{II}} \phi_{1} & \\
= & (1+E)(i k \operatorname{Re})^{-1} \\
& \times\left[\phi_{1}{ }^{\mathrm{IV}}-2 k^{2} \phi_{\mathrm{I}}{ }^{\mathrm{II}}+k^{4} \phi_{1}+a\left(\mathbf{U}^{\mathrm{IV}}-2 k \mathbf{U}^{\mathrm{III}}\right) e^{-k \eta}\right] \\
& +F_{1}\left(E^{\mathrm{I}}, E^{\mathrm{II}}, \phi^{\mathrm{I}}, \phi^{\mathrm{III}}\right),
\end{aligned}
$$

where Roman superscripts refer to differentiation with respect to curvilinear coordinate $\eta$ normal to wave surface, with boundary conditions

$$
\left.\begin{array}{rl}
\phi_{1}(0) & =a C \\
\phi_{1}^{\mathrm{I}}(0) & =-a \mathrm{U}^{\mathrm{I}}(0)
\end{array}\right\} .
$$

\section{Second order}

$$
\begin{gathered}
(\mathbf{U}-C)\left(\phi_{2}{ }^{\mathrm{II}}-4 k^{2} \phi_{2}\right)-\mathbf{U}^{\mathrm{II}} \phi_{2} \\
\quad+\frac{1}{2}\left(\phi_{1}{ }^{\mathrm{I}} \phi_{1}{ }^{\mathrm{II}}-\phi_{1} \phi_{1}{ }^{\mathrm{III}}\right)+F_{2}\left(\phi_{2}, \mathbf{U}^{\mathrm{IV}}, \mathbf{U}^{\mathrm{II}}\right) e^{-k \eta} \\
=(1+E)(2 i k \operatorname{Re})^{-1} \\
\quad \times\left[\phi_{2}{ }^{\mathrm{IV}}-8 k^{2} \phi_{2}{ }^{\mathrm{II}}+16 k^{4} \phi_{2}+F_{3}\left(\phi_{2}, \mathrm{U}\right) e^{-k \eta}\right] \\
+F_{4}\left(E^{\mathrm{I}}, E^{\mathrm{II}}, \phi^{\mathrm{I}}, \phi^{\mathrm{III}}\right),
\end{gathered}
$$

with boundary conditions

$$
\left.\begin{array}{rl}
\phi_{2}(0) & =k a^{2} C \\
\phi_{2} \mathrm{I}(0) & =-k a^{2} \mathbf{U}^{1}(0)-\frac{11}{2} k^{2} a^{2} C
\end{array}\right\} .
$$

It may be noted that the second-order equation is almost of the same type as the first-order equation with wavenumber $k$ replaced by $2 k$. In addition, there is a forcing term $\frac{1}{2}\left(\phi_{1}{ }^{\mathrm{I}} \phi_{1}{ }^{\mathrm{II}}-\phi_{1} \phi_{1}{ }^{\mathrm{III}}\right)$ due to self-interaction of the first-order solution. The boundary condition in the second-order problem is proportional to that of the first-order problem by a factor $k a$. The term $-(11 / 2)$ 
$\times\left(k^{2} a^{2} C\right)$ appearing in the second-order problem is usually a small quantity compared to the first term $-k a^{2} \mathrm{U}^{\mathrm{I}}(0)$. The terms $F_{1}$ to $F_{4}$ are found to be relatively unimportant during the calculations. Thus, the basic governing equation remains of the Orr-Sommerfeld type. One of the important physical quantities in windgenerated waves is the Reynolds stresses. Because the interface cannot support a stress discontinuity, this implies momentum transfer between wind and waves. If the Reynolds stress ( $-\overline{\text { wwi }}$ ) is positive, the wave is receiving momentum from the wind.

On the basis of the expansion procedure, we have

$\overrightarrow{u w}=i k\left(\phi_{1}{ }^{\mathrm{I}} \phi_{1}{ }^{*}-\phi_{1} \phi_{1}{ }^{\mathrm{I}}+2\left(\phi_{2}{ }^{\mathrm{I}} \phi_{2}{ }^{*}-\phi_{2} \phi_{2}{ }^{*}\right)+\cdots\right)$.

The term associated with $\phi_{1}$ is the Reynolds stress of the first harmonic, and the term associated with $\phi_{2}$ is the Reynolds stress of the second harmonic. Discussions of the Reynolds stresses distribution with respect to various parameters will be presented.

\section{Method of solution}

When the coefficients of the governing equation (15) vary in the range of interest, no closed form solution exists. However, a numerical integration is possible over the interval, by treating the boundary value problem as an initial value problem with two free parameters to be determined by the boundary conditions. The KuttaRunge fourth-order scheme has been used to integrate

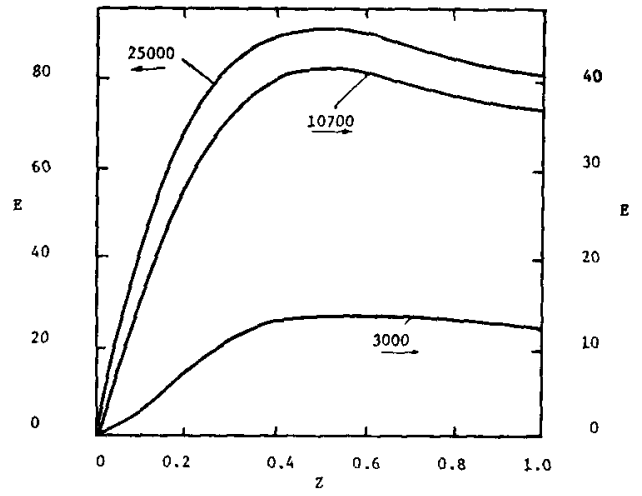

FIG. 2a. Eddy viscosity $E$ vs height $Z$.

the differential equation. A detailed description of the technique with application to hydrodynamic stability is given by Brown and Lee (1972).

In order to integrate the Orr-Sommerfeld equation, the mean turbulent wind profile $\mathbf{U}(z)$ must be specified. The profile used in this work made use of the eddy viscosity model of Cess (1958):

$$
\begin{aligned}
E(z)=\frac{1}{2}\left\{1+\frac{\kappa^{2} \operatorname{Re}^{2} R}{9}\right. & \left(2 z-z^{2}\right)^{2}\left(3-4 z+2 z^{2}\right)^{2} \\
& \left.\times\left[1-\exp \left(-\frac{\operatorname{Re} \sqrt{ } R}{K}-z\right)\right]^{2}\right\}^{\frac{1}{2}}-\frac{1}{2}
\end{aligned}
$$

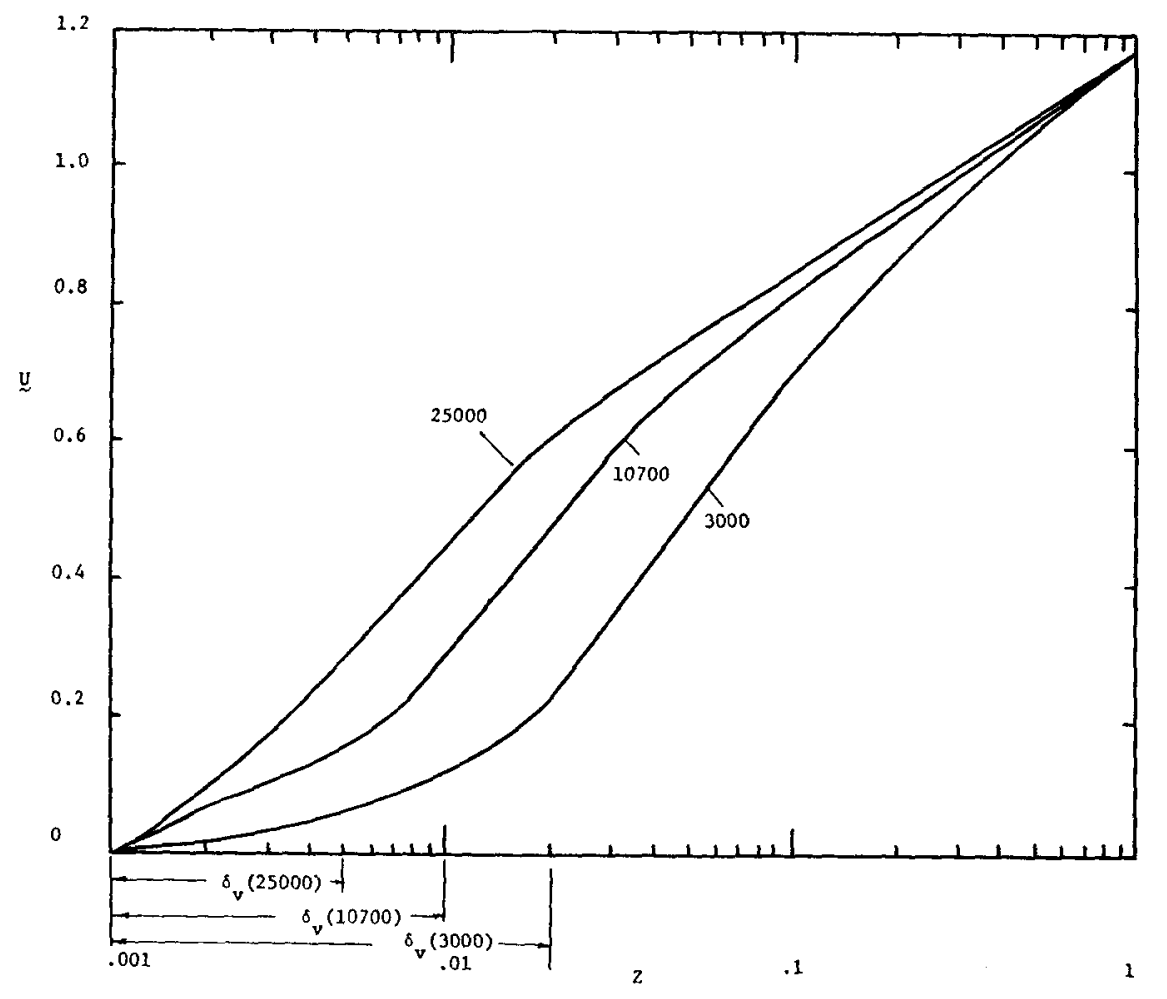

Fig. 2b. Mean wind velocity $U$ vs height $Z$ ( $\hat{o}_{v}$ viscous sublayer thickness). 
where $\kappa$ is the von Kármán constant having a value of $0.4, K$ is an empirical constant that was determined to have a value of 21 , and $R=(0.346 / 8) \sqrt{2} \mathrm{Re}^{\frac{1}{4}}$ is the average shear stress given by Cohen (1964). The wind velocity profile is then given by

$$
\mathrm{U}(z)=\operatorname{Re} R \int_{0}^{z} \frac{1-z}{1+E(z)} d z .
$$

The velocity profile is then bent to follow the wave profile, that is, $z$ is replaced by $\eta$ everywhere in the expression.

\section{Results}

Three Reynolds numbers $\operatorname{Re}=3000,10,700$ and 25,000 have been selected to demonstrate the influence of Reynolds number on wind-wave interaction. Both the eddy viscosity and the velocity profiles are presented in Fig. 2. It is of interest to note that the maximum eddy viscous Reynolds number varies from 220 at $\mathrm{Re}=3000$ to 280 at $\mathrm{Re}=25,000$. The form of the eddy viscosity $E(z)$ is prescribed such that a laminar layer is allowed next to the interface. Subsequently, the velocity gradient $\mathbf{U}^{\mathrm{I}}(0)$ is a linear function of the Reynolds number Re. The mean velocity profile shown in Fig. 2 is similar to that observed by Stewart during times when the waves were not present. It differs from the velocity profiles observed by Stewart during times when the waves were present. Such a discrepancy must exist if there is to be transfer of momentum from the air to wave because of a wave-induced Reynolds stress.

For wavenumber $k=1$, wave speed $C=0.5$, and wave amplitude $a=0.1$ and 0.2 , the Reynolds stresses of the fundamental as a function of the vertical distance $(Z)$ are presented in Fig. 3. Three features of the curves are of interest. First, at a fixed wave amplitude $(a=0.2)$, the maximum Reynolds stresses increase as the Reynolds numbers increase. This increase can be traced to the increase in $U^{\mathrm{I}}(0)$, which constitutes the viscous boundary conditions. It turns out that the viscous boundary condition $\phi_{1}^{\mathrm{I}}(0)=-a \mathbf{U}^{\mathrm{I}}(0)$ is dominant over the inviscid boundary condition $\phi_{1}(0)=a C$. Second, at

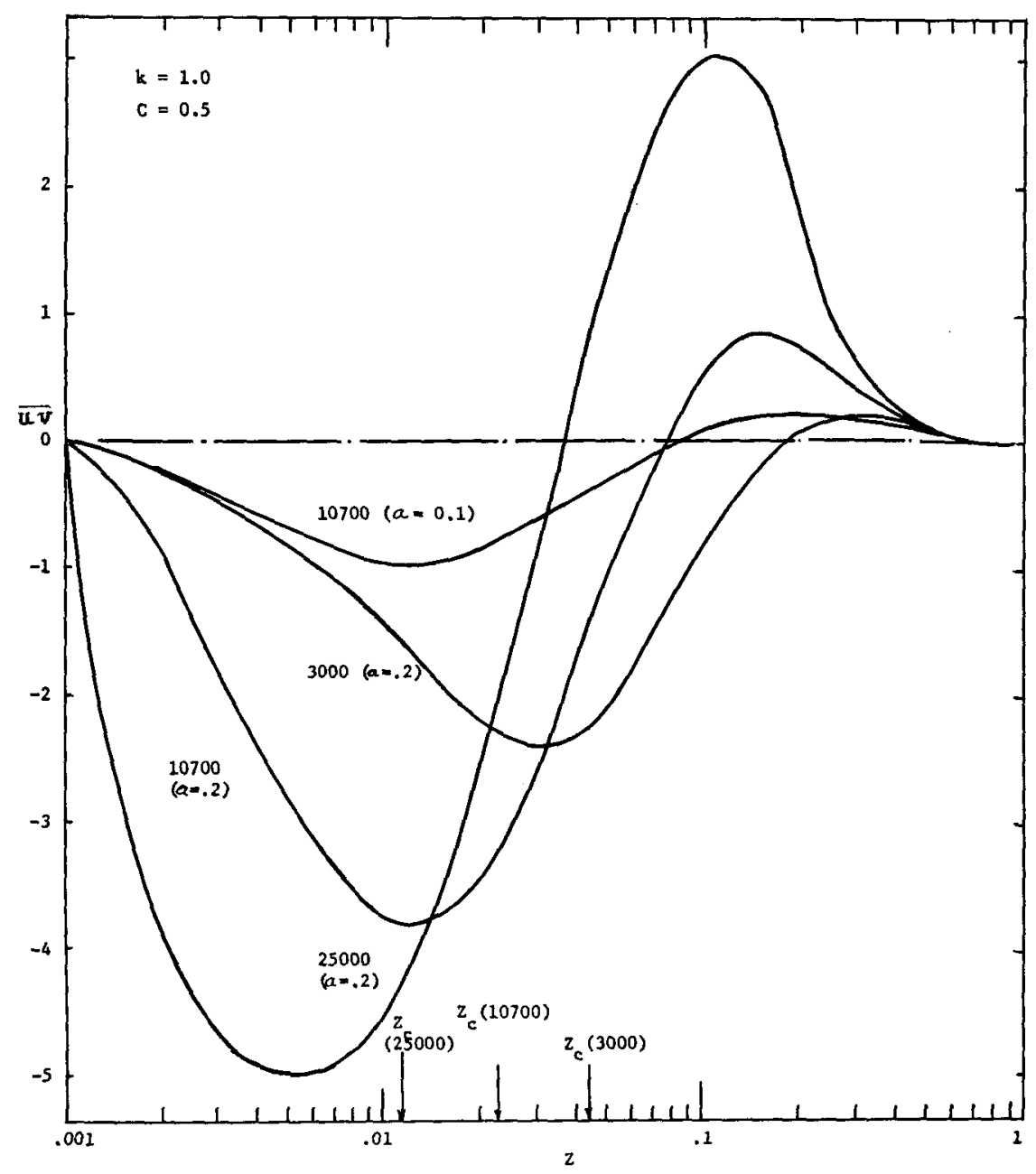

FIG. 3. Reynolds stresses vs height $Z$ (first harmonic). 


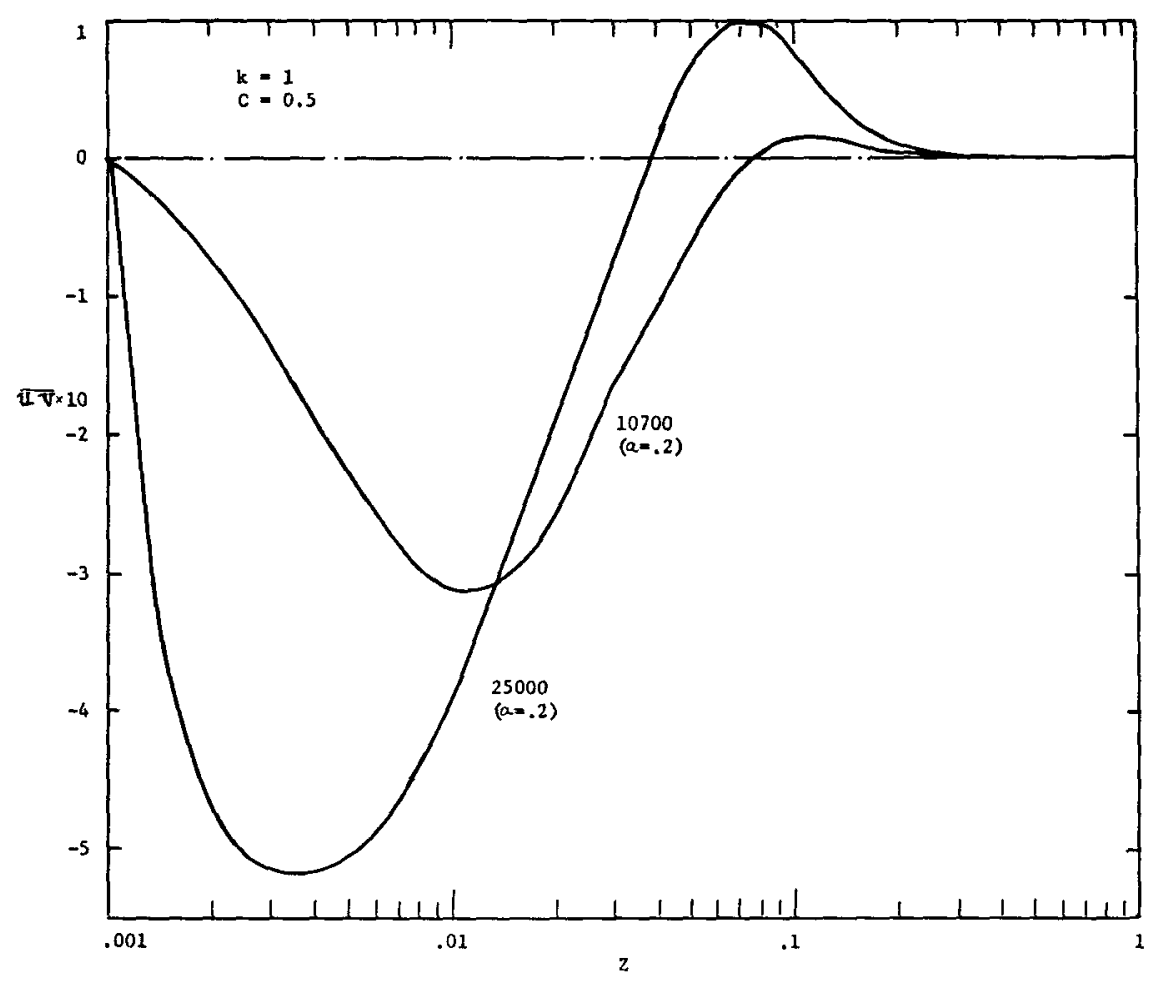

Fig. 4. Reynolds stresses vs height $Z$ (second harmonic).

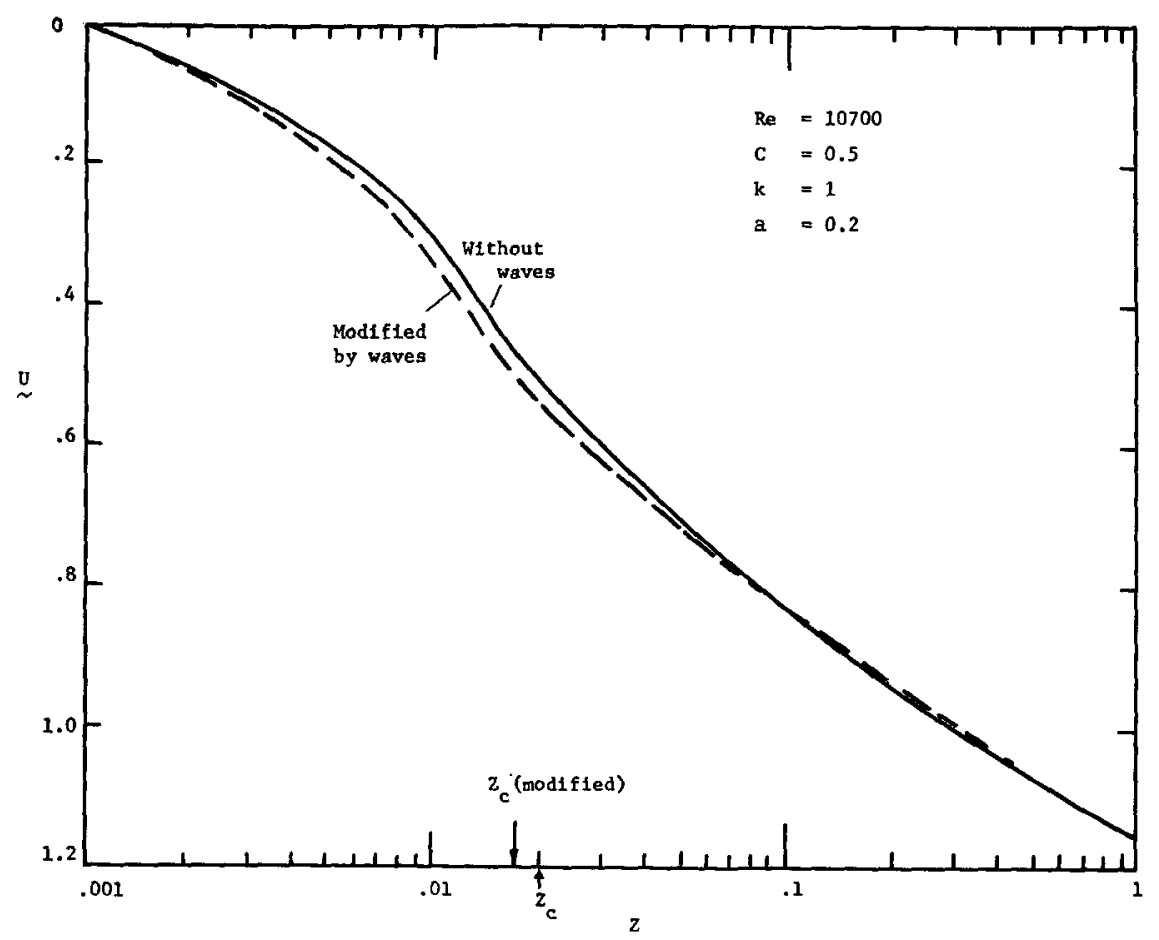

FIG. 5. Wind profile modified by waves. 
a fixed Reynolds number $(\operatorname{Re}=10,700)$, double in wave amplitude $a$ means quadruple in maximum Reynolds stresses. Justification of this behavior comes from the boundary conditions. Both $\phi_{1}$ and $\phi_{1}{ }^{\mathrm{I}}$ are of the order of the wave amplitude, $\mathrm{O}(a)$; then it follows that the Reynolds stresses are of the order of the square of the wave amplitude $\mathrm{O}\left(a^{2}\right)$. Third, we have also indicated in the figure the critical height variation for different Reynolds numbers. Increase in the Reynolds number implies lowering of the critical height and the maximum Reynolds stresses appear to occur at slightly lower height than the critical height.

The Reynolds stresses of the second harmonic are presented in Fig. 4 for $\mathrm{Re}=10,700$ and 25,000. Both of them are of the same shape as the first harmonic, with maximum about $8 \%$ of the first harmonic. By comparing the boundary conditions given by (16) and (18), we find that $\phi_{2}(0) / \phi_{1}(0)=k a$ and $\phi_{2}{ }^{1}(0) / \phi_{1}{ }^{1}(0)=k a$. The second equality is approximately valid because the term (11/2) $k^{2} a^{2} C$ which appeared in (18) is small compared to the term $k a^{2} \mathbf{U}^{\mathrm{I}}(0)$. Thus, the ratio between the second harmonic and the first harmonic will be approximately $2(k a)^{2}=0.08$. Agreements are found to be good for $\operatorname{Re}=10,700$. The ratio for $\operatorname{Re}=25,000$ is about $10 \%$.

Modification of the wind profile by Reynolds stress of the first harmonic is presented in Fig. 5. The modification is rather slight and the shift in critical height is also small. The change in the Reynolds stress due to the change of the wind profile would be only a small percent.

\section{Concluding remarks}

It is concluded that nonlinear contributions are twofold, namely the generation of higher harmonics and the modification of the wind profile. According to Reynolds (1968), the energy content of the second harmonic is about half that of the first harmonic attributed to Stewart's experiment. On the basis of the present results, this would require the product $k a$ to be of the order of $50 \%$. Certainly the perturbation procedure is not expected to work with perturbations of this magnitude. Therefore, the wind-wave interaction is an even stronger interaction phenomenon than predicted by the results of these perturbation analyses.

\section{APPENDIX}

\section{List of Symbols}

a wave amplitude

$C$ wave speed

$E$ eddy viscosity coefficient applied to the gradient of the perturbations

$g$ gravitational acceleration

$k$ wavenumber

$K$ an empirical constant

$P$ mean pressure

$p$ perturbation pressure

$q$ perturbed quantity $r_{i j} \quad$ wave-induced perturbation stress tensor

$R$ an empirical constant

$\operatorname{Re}$ Reynolds number

$s_{i j} \quad$ turbulent stress tensor perturbed by wave motion

$S_{i j}$ perturbation rate of strain tensor

$t$ time

$u \quad$ total velocity in $x$ direction

$u^{\prime} \quad$ turbulent velocity fluctuation in $x$ direction

$U$ perturbation velocity in $x$ direction

$\mathrm{U}$ mean velocity in $x$ direction

$v^{\prime} \quad$ turbulent velocity fluctuation in $y$ direction

$W$ perturbation velocity in $z$ direction

$w^{\prime}$ turbulent velocity fluctuation in $z$ direction

$x$ distance along the direction of wave propagation

$y$ distance along wave crest normal to wave surface

$z \quad$ distance normal to mean water surface

$Z$ an empirical constant

$\eta \quad$ curvilinear coordinate

$\zeta \quad$ water surface displacement from mean level

$\xi$ curvilinear coordinate tangent to wave surface

$\delta \quad$ boundary layer thickness (turbulent)

$\kappa \quad$ von Kármán's constant

$\rho \quad$ air density

$\phi$ streamfunction coefficient which is dependent only on $Z$

$\psi \quad$ streamfunction

〈) denotes averaging along $y$ direction

- (over bar) denotes averaging along $x$ direction

* denotes perturbation coefficient which is depen-

dent only on $Z$

* complex conjugate

Acknowledgments. The research reported herein was supported by the Oceanography Section, National Science Foundation, under Grant GA-26161.

\section{REFERENCES}

Benjamin, T. B. 1959: Shearing flow over a wavy boundary. J. Fluid Mech., 6, 161-205.

Brown, R., and F. Lee, 1972: A shooting method application to the hydrodynamic stability problem. J. Comput. Phys. (in press).

Cess, R. D., 1958: Westinghouse Research Report 8-0529-R24.

Cohen, L. S., 1964: Ph.D. thesis, Department of Chemical Engineering, University of Illinois.

Dobson, F. W., 1971: Measurements of atmospheric pressures on wind-generated sea waves. J. Fluid Mech., 48, 225-274.

Miles, J. W., 1957: On the generation of surface waves by shear flows. J. Fluid Mech., 3, 185-204.

Phillips, O. M., 1957: On the generation of waves by turbulent wind. $J$. Fhidd Mech., 2, 417-445.

Reynclds, W. C., 1968: The mechanics of an organized wave in a turbulent shear flow. Stanford University, National Science Foundation Progress Report (unpubl.).

Shemdin, O. H., 1969: Instantaneous velocity and pressure measurements above propagating waves. Tech. Rept. No. 4, NSF Grants GK-736 and GK-3986, College of Engineering, University of Florida.

Stewart, R. H., 1970: Laboratory studies of the velocity field over deep-water waves. J. Fluid Mech., 42, 733-754. 